\title{
Measurement of Double Differential Cross-Section for Electron Impact Ionization of Argon at $348 \mathrm{eV}$
}

\author{
G. Bozkurt, M. Yavuz, Z.N. Ozer And M. Dogan
}

Afyon Kocatepe University, Department of Physics, e-Col Laboratory, Afyonkarahisar, Turkey

In this study, we report the angular distribution of double differential cross sections of ejected electrons from Ar in intermediate energy region. Double differential cross-sections for electron impact ionization of argon have been measured at $348 \mathrm{eV}$. A hemispherical energy analyzer is used to analyze the ejected electrons with energies between 25 and $325 \mathrm{eV}$ and angles between $25^{\circ}$ and $130^{\circ}$.

DOI: 10.12693/APhysPolA.125.343

PACS: 34.80.Dp

\section{Introduction}

Renewed interest in measuring cross-sections for scattering electrons or positrons by noble gas atoms at intermediate energies has recently led to test various theoretical approximations. Ionization of the noble gases such as $\mathrm{He}, \mathrm{Ne}, \mathrm{Ar}, \mathrm{Kr}$, and Xe by electron impact is an important process in collision physics. Argon has been studied from the discharge point of view for more than 100 years and interest in this gas is due to several important fundamental and technical considerations. Cross-sections for electron-impact ionization have been measured and calculated since the early days of collision physics because of their importance to the kinetics and dynamics of collisions in relevance to many practical applications.

Previous experimental measurements of double differential cross-sections (DDCS) by electron impact for targets rather than helium are very few. The most itemized study is that of Opal et al. [1]. They presented DDCS data for several gases at different incident energies of a wide angular range. The literature contains the results from several experimental measurements on the double differential cross-section studies of Ar. The elastic scattering of electrons by noble gases has been extensively studied and a large number of either theoretically or experimentally obtained relative and absolute differential cross-sections are available at various incident electron energies and scattering angles. The critical minima appearing in Ar DCS spectra are the most sensitive test for comparison of experimental and theoretical results and, hence, give good possibilities for testing different theoretical models.

DuBois and Rudd [2] measured DDCS for 100-500 eV electron impact of $\mathrm{Ar}$ including $\mathrm{Ne}, \mathrm{N}_{2}$, and $\mathrm{H}_{2}$ data. Hippler et al. [3] and Chaudhry et al. [4] reported energy distributions of ejected electrons in double or (multiple) ionization of rare gases $300 \mathrm{eV}$ to $10 \mathrm{keV}$ electron impact. Another work is done by Santos et al. [5] at 500-750 and $1000 \mathrm{eV}$ electron impact single or multiple ionization DDCSs of Ar. Yates and Khakoo [6] have presented DDCSs of Ar for near threshold electron impact single ionization of Ar at 17, 18, 20, and $30 \mathrm{eV}$.

In this work, we used Ar target as a typical that is reasonable to expect to understand ionization mechanism of atomic systems. We have measured DDCS for asymmetric geometry for different kinematical conditions.

\section{Experimental setup}

The apparatus used to measure the DDCSs is described in Refs. [7-9]. Therefore the apparatus is described briefly here. The main components of the apparatus are two electrostatic hemispherical energy analyzers, an electron gun, the Faraday cup and the components for shielding interaction region and electronic control units for data acquisition. An electron beam produced by electron gun is directed into a gas target. Ejected electrons emitted at an angle of $\theta_{\mathrm{b}}$ are energy analyzed by a hemispherical electrostatic analyzer and detected by a channel electron multiplier (CEM). DDCSs for Ar were measured for selected ejected electron energies by manually adjusting the analyzing voltage and recording the scattered signals [8] (Fig. 1).

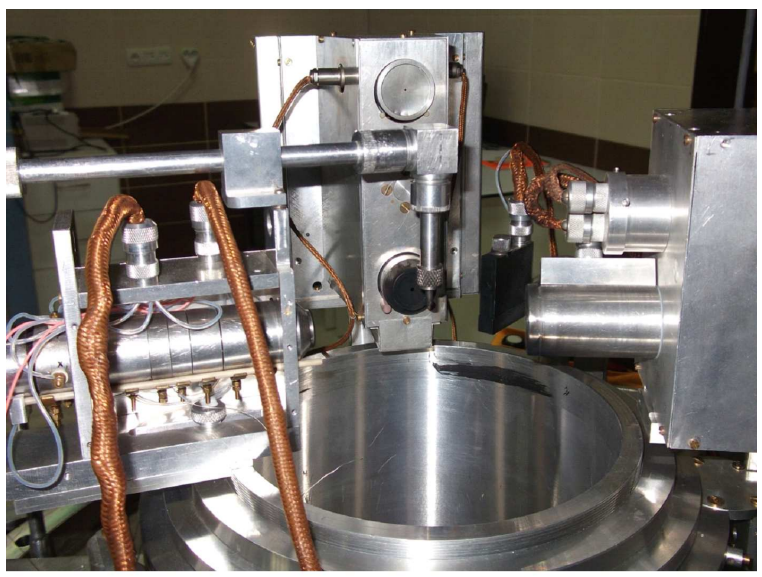

Fig. 1. Picture of electron spectrometer.

The electron beam was produced by an electron gun that during the present measurements has been operated at $348 \mathrm{eV}$ energy and at currents about $0.2 \mu \mathrm{A}$. The diameter of the electron beam at the scattering center was $\approx 2 \mathrm{~mm}$. The electron beam current was continuously monitored by a Faraday cup. The effusive gaseous beam 
was let into the chamber via a needle orthogonal to the electron beam.

\section{Results and discussion}

We have carried out measurement of elastic differential cross-section (DCS) before performing DDCS measurements (Fig. 2). DCS suffers drastic change versus both incident electron energy and scattering angle in the region close to the critical points. Measured elastic DCS shows deep minima at $\approx 100^{\circ}$. This structure is a well-known high angle position in literature 10, 11].

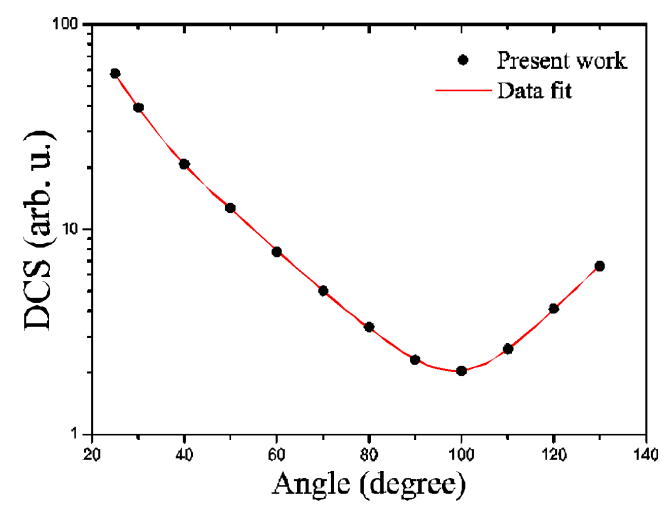

Fig. 2. Elastic differential cross-section measurement of argon by electron impact with $348 \mathrm{eV}$.

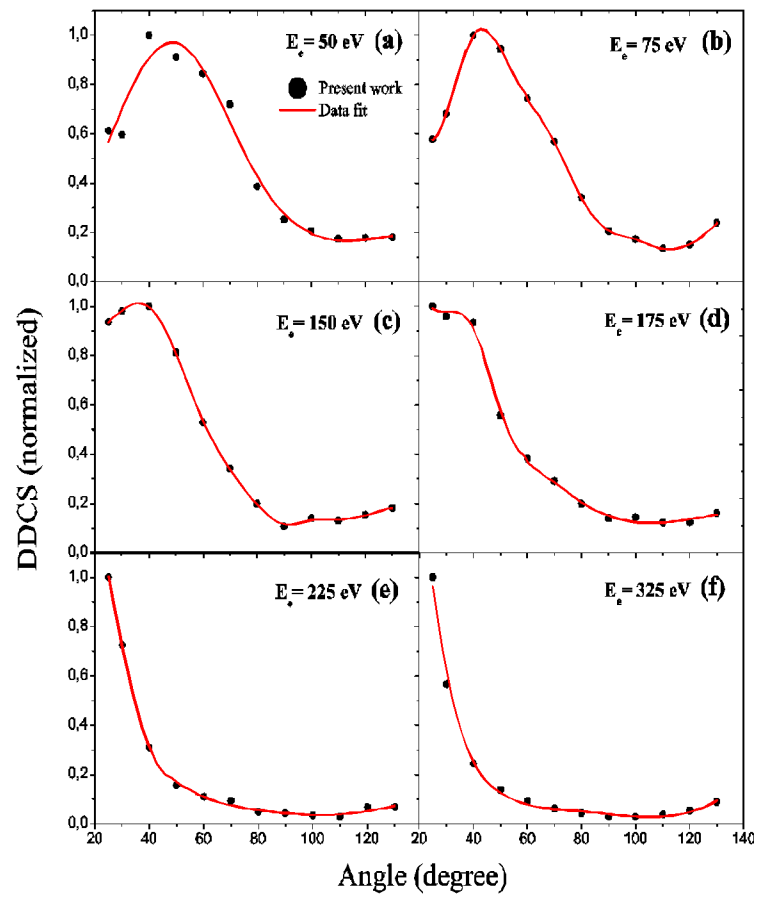

Fig. 3. Measured DDCS results of Ar for $348 \mathrm{eV}$ electron impact energy at (a) 50, (b) 75 , (c) 150, (d) 175, (e) 225 , (f) $325 \mathrm{eV}$ ejected electron energies.
This kind of comparison is more sensitive to experimental procedures, so the comparison of DCSs as a function of scattering angle is a tough test for the reliability of the spectrometer.

DDCSs for single ionization of argon by $348 \mathrm{eV}$ electron impact have been measured for ejection energies 50 , $75,150,175,225$, and $325 \mathrm{eV}$. Ejection angles were in between $25^{\circ}$ and $130^{\circ}$. Figure 3 shows the DDCS results of Ar.

The results show maxima and this structure has a systematic variation with energy. As the ejection energy increases, the position of the maxima shifts towards smaller angles. The forward peak attributed to a binary collision between incident electron and atomic electron can be shown in Fig. $3 \mathrm{a}-\mathrm{c}$ at relatively small angles. So, a binary collision can be commented in these kinematic conditions. A smooth change is observed at higher ejection energies (Fig. 3e and c). It is possible that maxima of the binary collision shifted to much smaller angles than experimental range.

\section{Acknowledgments}

This work is supported by TÜBİTAK under grant No. 109 T738 and by AKU BAPK under grant No. 12.FENBIL.12.

\section{References}

[1] C.B. Opal, E.C. Beaty, W.K. Peterson, At. Data 4, 209 (1972).

[2] R.D. Dubois, M.E. Rudd, Phys. Rev. A 17, 843 (1978).

[3] R. Hippler, K. Saeed, I. McGregor, H. Kleinpoppen, Z. Phys. A 307, 83 (1982).

[4] M.A. Chaudhry, A.J. Duncan, R. Hippler, H. Kleinpoppen, Phys. Rev. A 39, 530 (1989).

[5] A.C.F. Santos, A. Hasan, T. Yates, R.D. DuBois, Phys. Rev. A 67, 052708 (2003).

[6] B.R. Yates, M.A. Khakoo, Phys. Rev. A 83, 042712 (2011).

[7] Z.N. Ozer, H. Chaluvadi, M. Ulu, M. Dogan, B. Aktas, D. Madison, Phys. Rev. A 87, 042704 (2013).

[8] M. Dogan, M. Ulu, O. Sise, J. Electron. Spectrosc. Relat. Phenom. 161, 58 (2007).

[9] M. Dogan, M. Ulu, Z.N. Ozer, M. Yavuz, G. Bozkurt, J. Spectr. 2013, 192917 (2013).

[10] J.E. Sienkiewicz, S. Telega, P. Syty, S. Fritzsche, $R a-$ diat. Phys. Chem. 68, 285 (2003).

[11] A.R. Milosavljevic, S. Telega, D. Sevic, J.E. Sienkiewicz, B.P. Marinkovic, Radiat. Phys. Chem. 71, 1015 (2004). 